\title{
Ensuring timely repair of power transformers based on data of complex diagnostics
}

\author{
Makhsud Sultanov ${ }^{1, *}$,Elena Zenina ${ }^{1}$, Peter Shamigulov ${ }^{1}$, Valentina Lunenko ${ }^{1}$, and Olga Zhelyaskova ${ }^{1}$ \\ ${ }^{1}$ National Research University "MPEI", Power Engineering Department, 404110 Volzhsky, Russia
}

\begin{abstract}
Timely diagnosis of power transformers is an essential component of ensuring reliable and safe operation of power stations and substations, on which the reliability of the power system depends. Detection of defects in the initial stage allows to maintain reliable operation of transformers, helps to define the "life cycle" and simplify the planning of their replacement. The paper presents an analysis of existing approaches to the creation of power equipment diagnostics systems using the example of power transformers. A neural network model has been developed, demonstrating the possibility of using power transformers to estimate the current residual resource based on the analysis of available diagnostic data.
\end{abstract}

\section{Introduction}

At present special attention is paid to creation of all conditions of normal accident-free and efficient operation of electrical equipment of electro-energy system (EES) [1]. Power transformers, as one of the main equipment of stations and substations, are quite reliable. However, the failures of transformers with an operating time of more than 25 years are about $33 \%$. The actual condition of the transformer can be determined by means of a comprehensive diagnostic examination, which is a time-consuming and expensive procedure. For the purposes of strategic repair planning, it is enough to have averaged data on the reliability characteristics of a certain set of transformers. Detection of defects in the initial stage allows you to maintain reliable operation of transformers, helps to define the "life cycle" and simplify the planning of their replacement. An effective program of diagnostic tests reduces the likelihood of defects and failure of transformers. Diagnostics of the transformer condition is complex, it is carried out at the stage of transformer manufacture, before it is put into operation and during operation.

In general, to assess the technical condition of the transformer, the following measures are carried out [2]:

- Analysis of technical features of transformers (main parameters of the transformer, results of acceptance tests of the transformer at the manufacturer, main parameters and results of the Llive-Line Working (LLW) tests, main parameters and results of the input tests, identification of areas with minimal reserves of electrical strength of insulation, assessment of mechanical resistance of windings in case of short circuit, evaluation of temperature distribution in operating modes and identification of the most heated zones, analysis of operating experience of transformers of this type and transformers of similar design);
- Analysis of transformer operating conditions (analysis of operating modes, results of preventive tests and determination of characteristics that differ from the norms, analysis of faults detected during operation, their nature and methods of elimination, external inspection of the transformer);

- Tests and checks at the operating transformer (assessment of possible reduction of electrical strength of insulation, check of oil temperature expansion compensation systems functioning, determination of partial discharges (PD) presence, assessment of possible reduction of compaction forces of windings and magnetic conductor, measurement of vibration characteristics of cooling system elements, measurement of magnetic field strength along the perimeter of the tank at the place of its connector, check of oil leak absence in the tank, check of thermal annunciators' operation, phase measurement of current, oil pumps consumption of the cooling system, oil testing, thermal imaging control of the transformer tank, inlets, tank of LLW device, elements of cooling system, measurement of current in neutral and in transformer grounding, measurement of oil flows in the cooling system, evaluation of overheating of the tank, external structures and bushings by the method of thermal imaging control, measurement of the characteristics of the bushings at operating voltage);

- Tests after disconnection and disengagement of the transformer (measurement of idling losses and magnetization current, measuring the insulation characteristics of windings, measuring the insulation characteristics of bushings, measuring the DC resistance of windings, assessment of the state of the LLW contactor, assessment of the kinematics state of the LLW device, measurement of short-circuit resistance, oil sampling, sampling and determination of the degree of polymerization of paper insulation, transformer tightness

\footnotetext{
Corresponding author: opp77@bk.ru
} 
test, inspection and check of gas relay condition, terminal box, LLW device drive equipment, thermal annunciators, check of insulation resistance of stator windings of oil pumps motors).

\section{Analysis of existing approaches to power equipment diagnostics systems}

Based on the development of foreign and domestic systems for continuous diagnostic monitoring of power transformers, it is possible to distinguish several transformer systems necessary for their control: insulation, cooling, current-carrying part; magnetic core, etc. (LLW, Corps...). With the help of automated diagnostic systems, measured values are compared with normal operational parameters, detected defects and deviations of parameters from standard values are monitored. As a result, the intelligent system, in accordance with the logic algorithm embedded in it, outputs the measurement result with a certain degree of its processing.

For the first time, a comprehensive automated system for monitoring the state of power transformers was developed in the United States. The TPAS (Transformer Performance Analysis System) monitors to identify the maximum possible variants of defects at an early stage of their development [1].

This system reveals the following defects of the working transformer: partial breakdown of insulation, reduction of mechanical strength due to short circuits, places of overheating of windings and magnetic conductor, damage to LLW and bushings, and also controls the level of vibration, moisture and gases in oil, heating temperature of windings. The TPAS system monitors all transformer components through current and voltage sensors and transformers. The system is based on the concept of Model Based Monitoring [2]. Development of improvement of control sensors is underway.

Siemens from Germany also developed a continuous transformer condition monitoring system based on a modular principle with the replacement of individual modules. This system consists of a set of sensors, analog-to-digital converters and a computer part. The developers offer two configuration options:

- complex of equipment and computer, located in one cabinet;

- the equipment complex is installed on the transformer tank, and the computer is located in the control room.

The computer performs the functions of processing and archiving data, evaluating the results. Proposed control system is designed for transformers from 10 to 100 MVA [3]. In the latter version, Siemens supplies the Sitram + second generation continuous monitoring system based on the Sitnatic computing system. It receives signals from sensors installed on the transformer, which processes, analyzes and visualizes measurement data. The system provides early detection of defects in the transformer and notification of this on the control panel.
ABB Secheron from Switzerland does not lag behind the development of a continuous control system for power transformers. This system is proposed for transformers with a capacity of 100 MVA. Proposed system applies comparison of measured parameters with processes obtained on mathematical models in transformer. Similar to the previous systems, a sensor for each element is installed in the transformer. Then the acquisition and processing of the received data is carried out by the T-MAP 230. Such a system not only collects data, but also captures relays and overloads [4].

"Energy Automation" LLC offers its system of continuous control of the SAFE-T power transformer to the domestic manufacturer. This system complies with the requirements of transformer equipment diagnostics presented by OJSC FGC UES.

A comprehensive monitoring and diagnostics system of the TDM brand (Transformer Diagnostics Monitor) is developed and manufactured by the Russian company DIMRUS. TDM is widely known in the domestic market of diagnostic systems. This system allows you to monitor the compliance of the current parameters of the transformer with regulatory requirements; perform automated expert diagnostics of defects and evaluation of the transformer technical condition and transfer it to the higher level APCS-TP system for use in systems for monitoring the state of process units and process chains of electric power transmission and conversion.

The system uses a SCADA system and proprietary software with a multi-level interface, which currently provides information about the transformer under investigation in different types (diagram, graphs, tables). Control can be carried out remotely via the Internet.

To increase diagnostic efficiency, the system uses mathematical algorithms, according to which parameters are calculated on the basis of primary data from sensors, due to which faults and defects are more accurately determined [5]. Also known is a specialized system for partial evaluation of technical equipment of electrical networks [6].

Recently, a data processing technique, fixed by order of the Ministry of Energy of Russia [7], has become widespread, which allows calculating weight coefficients that determine the technical condition of the equipment being diagnosed. According to a fairly wide range of types and parameters of the main process equipment and its functional parts from generators to power lines, depending on the composition, type and cost, this system determines a formalized indicator in the form of a technical condition index (TCI). According to TCI a conclusion is made on the operability of the equipment and the need to put it out for repair.

Analysis of the given diagnostic systems shows that all tasks of operation control of the main process equipment under the traditional decision-making procedure are divided into three main groups:

- The first group includes operational decisions on ongoing repairs;

- The second group includes issues related to the determination of the time for major repairs or modernization with a long withdrawal of equipment from work; 
- The third group includes issues related to technical and economic justification of equipment decommissioning.

\section{Development of neural network model for estimation of current residual life of power transformers}

The complex analysis of estimation parameters of technical condition of power transformers is complicated by the fact that the combined effect of different combinations of indicators on the value of the actual residual resource of the equipment does not have sufficient statistical assessment for each type of transformer. Since the failures of transformers occur quite rarely and the accumulated complex diagnostic data, due to which the failure occurred, are not statistically significant.

To solve this problem, a combined analysis of diagnostic data accumulated for various types of transformers can be applied with the output of an estimate of the calculated residual resource for a particular type of transformer. This analysis can be used as a recommendation to plan the transformers for repair or maintenance.

To implement the above approach, it is possible to use neural network mathematical models, due to which it is possible to process a large number of heterogeneous input data. Training of neural network on templates, formed on examples of diagnostic sets for different types of equipment, will allow to identify the most common factors affecting the value of residual resource of equipment.

To develop a mathematical model, artificial data were generated in three versions - as reducing the resource of equipment, as not affecting the resource and as increasing the residual resource. The template developed in this way will allow for real diagnostic data of power transformers to form a training template corresponding to the real qualitative impact on the resource, choosing one of three versions of the mathematical model corresponding to the nature of the actual initial data.

Simulation of diagnostic data was carried out using the example of 500 transformers, of which a failure (accident, unplanned shutdown) was simulated for 50 before the next overhaul or maintenance period. Orange data analysis and visualization tool [8] was used to detect stable patterns in test data.

Consider three versions of the mathematical model of the influence of the amount of electrical energy transferred for the period after commissioning on the value of the change in the residual life of the transformer (Figure 4). The graphs show the simulation of the time remaining at the time of failure until the transformer is scheduled for repair against the amount of transferred energy.

We express the amount of transmitted energy with a dimensionless coefficient determined by:

$$
P^{\prime}=\frac{1000 \cdot S}{S_{\text {HOM }} \cdot 24 \cdot 365}
$$

where $\mathrm{S}$ - the amount of energy transferred by the transformer, $\mathrm{kW} \cdot \mathrm{hr}$;

$\mathrm{S}_{\text {nom, }}$ - transformer rated power, MVA.

The denominator of the expression (1) is the amount of energy transferred by this transformer per year, subject to continuous round-the-clock loading at rated power.

Three variants of the mathematical model of the influence of the amount of electrical energy transferred during the period after commissioning on the amount of change in the residual life of the transformer are considered.

We will compose data sets simulating the influence (or absence of influence) on the residual life of power transformers accumulated during operation:

1. Thermal action of the actuated current of the transformer in the form of a time accumulating sum of the current square;

2. Storage sum of square of reduced voltage of transformer;

3. Accumulation sum of voltage square of stator zero sequence (for transformers with isolated neutral on at least one of the sides);

4. Cumulative sum of relative deterioration of insulation of bushings $\left(\frac{R_{\text {isol.nom }}}{R_{\text {isol }}}\right)^{2} \cdot t$ (if there is an insulation monitoring system for the bushings);

5. Accumulation sum of square of absolute insulation temperature (oil, solid insulation);

6. Number of inclusions

7. Number of LLW actuations;

8. The number of loops in the system to be powered;

9. Cumulative sum of short circuit current values;

10. The number of thunderstorms;

11. Number of switching overvoltage;

12. Accumulative sum of switching overvoltage values .

The values of currents and voltages given in paragraphs 3, 9, 12 are determined as relative values with respect to the nominal values of current and voltage.

For all 12 parameter sets, test data sets were generated in a similar manner to the data on the amount of electrical energy transferred for the period after commissioning (shown in Figure 5).

The listed 12 criteria and dimensionless coefficient of the amount of energy transferred (the 13th criterion) form a universal set of the considered criteria, which can be implemented on any transformer regardless of its model.

In each of the 13 sets of test data, three possible qualities of influence on the value of the residual life of the transformer are provided - neutral, negative and positive. Each set is supposed to have 50 transformers with failure and 450 transformers successfully spent overhaul interval. The resulting set of test data can 
generate $3^{13}=1.594 .323$ different combinations that can be used to train the neural network to estimate the residual life of power transformers.

As an example, consider one training template in which each of the 13 parameters for 50 transformers has a negative character in terms of residual resource.

The result of comparing the determination of the residual resource using the neural network with the initial data is shown in Figure 1.

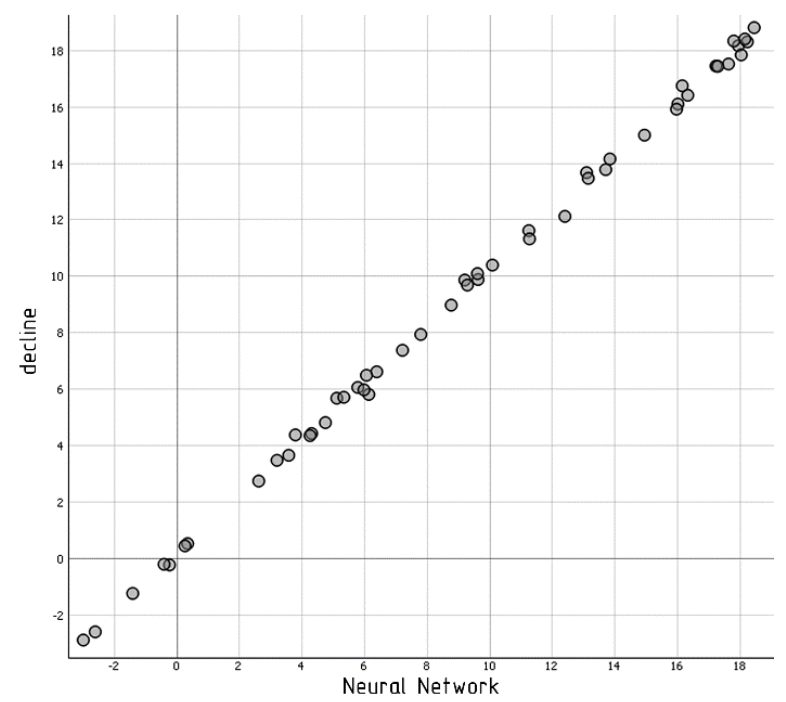

Fig. 1. Comparison of the results of residual resource determination performed by Neural Network and data from training template (decline).

Figure 2 shows the dependence of the residual life of 450 transformers depending on all 13 parameters, using the neural network.

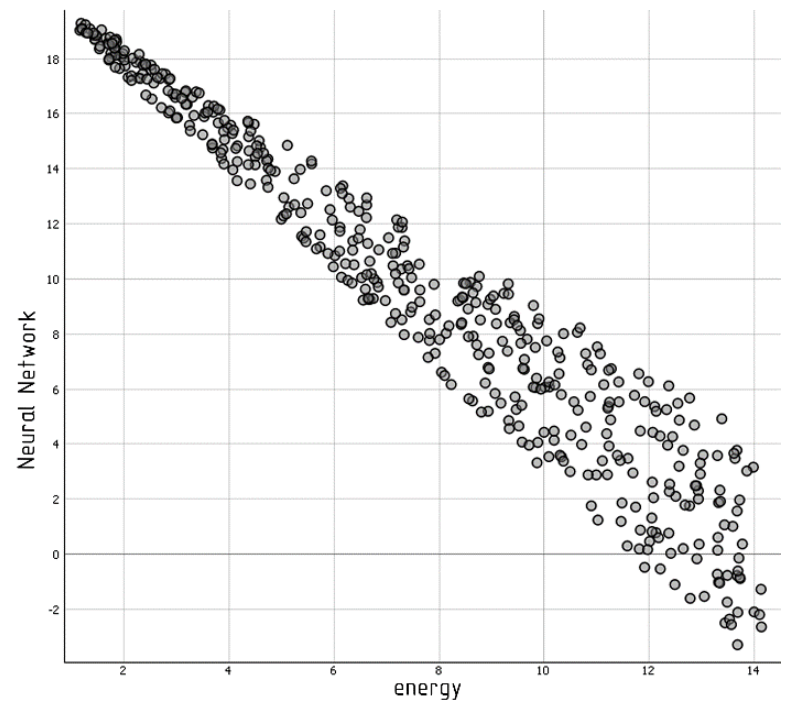

Fig. 2. The dependence of the residual resource predicted by the neural network (in years) on relative energy transfer.

The analysis of the data presented in Figure 8 suggests that with a small amount of transferred energy, the residual life of the transformer is weakly dependent on other factors, since the predicted values are grouped in the range of 20 years. This is because most parameters have a cumulative (integral) nature over time. However, with the increase in the operating time of the transferred transformer energy, the influence of other factors becomes more noticeable, as evidenced by the increasing spread of predicted values.

\section{Conclusions}

Thus, the developed neural network model demonstrates the possibility of its application for estimating the current residual life of power transformers based on the analysis of diagnostic data. At the same time, diagnostic data of transformers of various types can be used as training templates, which increases statistical reliability of simulation results. Equations should be centred and should be numbered with the number on the right-hand side.

The research is funded by Russian Federation public contract № FSWF-2020-0025 "Technique development and method analysis for ensuring power system object security and competitiveness based on the digital technologies".

\section{References}

1. A.G. Klimov, V.R. Kozlov, Diagnostic equipment and non-destructive equipment for energy facilities, Ener., 1, 42 (2013)

2. B.A. Alekseev, Large power transformers: state control at work and during revision, Energoprogress, 88, (2010)

3. B.A. Alekseev, Systems of continuous monitoring of the state of large power transformers, NC ENAS (2009)

4. E.G. Dashevsky, Automated system for monitoring the technical condition of power transformers with a long life, Dissertation for the degree of Ph.D. (2011)

5. Energy Automation LLC [Electronic resource] Available at: http://www. enera.com.ua/products/production/Safe-t/

6. V.P. Kuznetsov, V. Zhdanov, N.S. Ovchinnikova, G.G. Yudin, Automated system for assessing the technical condition of electrical equipment [Electronic resource] Available at: https://forca.ru/knigi/arhivy/arhivy-2001-2.html

7. Evaluation methodology of technical condition of the main process equipment and power transmission lines of electric stations and electric networks and determination of the optimal type, composition and cost of technical impact on the equipment [Electronic resource] Available at: https://www.garant.ru/products/ipo/prime/doc/56 619202

8. Algorithm for identifying obvious defects of technological equipment in the energy sector based on the neural network model, Scientific Gazette of NSTU, 63 (2), 70-89 (2016) 